\title{
Hybrid TW-TOA/TDOA Positioning Algorithms for Cooperative Wireless Networks
}

\author{
Mohammad Reza Gholami ${ }^{\dagger}$, Sinan Gezici ${ }^{\sharp}$, Erik G. Ström ${ }^{\dagger}$, and Mats Rydström ${ }^{\dagger}$ \\ $\dagger$ Chalmers University of Technology, Department of Signals and Systems, Gothenburg, Sweden \\ $\sharp$ Bilkent University, Department of Electrical and Electronics Engineering, Ankara, Turkey
}

\begin{abstract}
The problem of positioning an unknown target is studied for a cooperative wireless sensor network using hybrid two-way time-of-arrival and time-difference-of-arrival measurements. A maximum likelihood estimator (MLE) can be employed to solve the problem. Due to the non-linear nature of the cost function in the MLE, a numerical method, e.g., an iterative search algorithm with a good initial point, should be taken to accurately estimate the target. To avoid drawbacks in a numerical method, we instead linearize the measurements and obtain a new two-step estimator that has a closed-form solution in each step. Simulation results confirm that the proposed linear estimator can attain Cramér-Rao lower bound for sufficiently high SNR.
\end{abstract}

Index Terms- Cooperative positioning, linear estimator, wireless sensor networks.

\section{INTRODUCTION}

Positioning information is a vital requirement for every wireless sensor network (WSN). Most studies in the literature assume that there are some reference nodes, also called anchor nodes, that can be used to estimate the position of an unknown target node [1]. In general, there are various positioning algorithms based on time-of-arrival (TOA), time-difference-ofarrival (TDOA), received signal-strength , and angle-of-arrival that can be used in different applications [1].

Two-way TOA (TW-TOA) has been considered as an effective approach in the literature (e.g., [2]), mainly because of its relatively high accuracy and lack of synchronization requirements. In this approach, a reference node sends a signal to a target node, and waits for a response from it. The roundtrip time delay between the reference node and the target node gives an estimate of the distance between them. As the number of reference nodes in a WSN increases, the position of the target node can be estimated more accurately via TWTOA estimation. Since, in practice, there are some limitations on increasing the number of reference nodes due to power and complexity constraints, the idea of cooperation between reference nodes is proposed in [3] to decrease the number of transmissions, and its theoretical analysis is presented in [2]. In this method, some reference nodes, called primary reference nodes (PRNs), initiate range estimation by sending a signal. The target replies to received signals by sending an acknowledgement. Suppose that there are some other reference nodes, which can listen to both signals, and are called as

This work was supported in part by the European Commission in the framework of the FP7 Network of Excellence in Wireless COMmunications NEWCOM++ (contract no. 216715) and in part by the Swedish Research Council (contract no. 2007-6363) secondary reference nodes (SRNs). It has been shown that the SRNs can help the PRNs to estimate the target position more accurately [2]. In fact, it is possible to get the same performance with fewer PRNs when measurements from the SRNs are involved in the positioning process. The model considered here is based on cooperation between reference nodes, which is different from targets' cooperation in a cooperative network [4].

In this work, it is assumed that the SRNs are able to receive signals from both the target and the PRNs. Therefore, the SRNs are able to measure the TDOA between the target signal and the signals of the PRNs. In this case, a maximum likelihood estimator derived in [2] can be employed to improve the positioning accuracy compared to the non-cooperative approach. However, due to the non-linear nature of the cost function in the MLE, a numerical method, e.g., an iterative search algorithm with a good initial point, should be used to obtain an accurate estimate of the target's position. There might be some drawbacks in using a numerical method in practice. For example high complexity or convergence problem can limit the use of the numerical methods. To avoid drawbacks in numerical method, using two different linearization techniques we obtain a two-step linear estimator. In the first step, using a non-linear pre-processing similar to some previous work for conventional networks, a coarse estimator is obtained. In the second step, using the first step estimation, another linear set of measurements different to the first step is derived. The secondstep estimator is a regularized least squares which refines the first estimate. In addition, in every step, a closed-form solution is derived. Simulation results show that for sufficiently large SNR, the estimator attains the Carmér-Rao lower bound (CRLB).

The remainder of the paper is organized as follows. Section II explains the system model considered in this paper. The linear estimator is derived in Section III and simulation results are discussed in Section IV. Finally Section V makes some concluding remarks.

\section{SySTEM MODEL}

Let us consider a two-dimensional network ${ }^{1}$ with $N+$ $M$ reference nodes located at known positions, $\mathbf{x}_{i}=$ $\left[\begin{array}{ll}x_{i, 1} & x_{i, 2}\end{array}\right]^{T} \in \mathbb{R}^{2}, i=1, \ldots, N+M$. Suppose that $N$ PRNs

\footnotetext{
${ }^{1}$ The generalization to a three-dimensional scenario is straightforward, but is not explored here.
} 
are used to measure the TW-TOA between the PRNs and the target to be located and that $M$ SRNs are able to listen and measure signals transmitted by the PRNs and the target. For simplicity, we assume that the first $N$ sensors are the primary nodes and the remaining $M$ sensors are the secondary nodes.

Let $\mathcal{C}=\{(i, j) \mid$ PRN $i$ and SRN $j$ are connected $\}$ be the set of all pairs with one primary node and one secondary node which are connected. In this paper for simplicity, we assume a fully connected network. The TW-TOA measurement between primary node $i$ and the target, located at coordinates $\boldsymbol{\theta}=$ $\left[\begin{array}{ll}\theta_{1} & \theta_{2}\end{array}\right]^{T} \in \mathbb{R}^{2}$, can be written as [2]

$$
\hat{t}_{i}=\frac{r_{i}}{c}+\frac{\tilde{n}_{T, i}}{2}+\frac{\tilde{n}_{i, T}}{2}, \quad i=1, \ldots, N,
$$

where $c$ is the speed of propagation, $r_{i}=\left\|\mathbf{x}_{i}-\boldsymbol{\theta}\right\|$ is the distance between the $i$ th PRN and the point $\boldsymbol{\theta}, \tilde{n}_{i, T}$ is the TOA estimation error at the target node for the signal transmitted from the $i$ th PRN, and $\tilde{n}_{T, i}$ is the TOA estimation at the $i$ th PRN for the signal transmitted from the target node. The estimation errors are modeled as zero-mean Gaussian random variables with variances $\sigma_{T, i}^{2} / c^{2}$ and $\sigma_{i, T}^{2} / c^{2}$; i.e., $\tilde{n}_{T, i} \sim \mathcal{N}\left(0, \sigma_{T, i}^{2} / c^{2}\right)$ and $\tilde{n}_{i, T} \sim \mathcal{N}\left(0, \sigma_{i, T}^{2} / c^{2}\right)[2]$.

Suppose that the SRNs are able to measure the TOA based on the received signal from the target and the PRNs. The TOA estimate of the $i$ th PRN in the $j$ th SRN is

$$
\hat{t}_{i, j}=T_{o_{i}}+\frac{r_{i, j}}{c}+\tilde{n}_{i, j}, \quad(i, j) \in \mathcal{C},
$$

where the $i$ th PRN sends its signal at time instant $T_{o_{i}}$, that is unknown to the SRN, $r_{i, j}=\left\|\mathbf{z}_{i}-\mathbf{z}_{j}\right\|$ is the distance between primary node $i$ and secondary node $j$, and $\tilde{n}_{i, j}$ is modeled as a Gaussian random variable $\tilde{n}_{i, j} \sim \mathcal{N}\left(0, \sigma_{i, j}^{2} / c^{2}\right)$. Suppose that the response signal from the target to this signal is also received by the $j$ th SRN. The TOA estimate for this signal is given by

$$
\hat{t}_{i, T, j}=T_{o_{i}}+\frac{r_{i}}{c}+\frac{r_{j}}{c}+\tilde{n}_{i, T}+\tilde{n}_{T, j}, \quad(i, j) \in \mathcal{C} .
$$

Having these two measurements in the SRN, namely, measurement in (2) and in (3), we are able to measure the TDOA between the $i$ th PRN and the target which corresponds to the distance from the $i$ th PRN to the target plus the distance from the target to the $j$ th SRN.

\section{Positioning Algorithms}

To gain some insight into the problem, let us consider Fig. 1(a), where one PRN performs TW-TOA estimation with the target. Namely, the PRN sends a signal to the target, and the target replies to this signal. Here, we assume that either an estimate of the turn-around time is available [2] or the turnaround time is extremely small such that it can be neglected. Suppose that two other nodes (SRN1 and SRN2) listen to both signals. Since the distances between the reference nodes are known, it is possible in the secondary node to estimate the time reference from (2); Hence, the SRNs are able to estimate the overall distance from the PRN to the target and the target
$\mathcal{C}_{1}=\{(1,2),(1,3)\}$

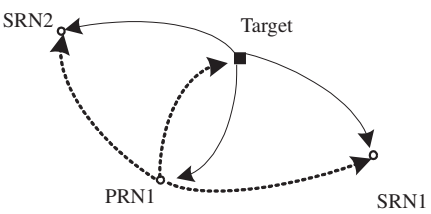

(a)

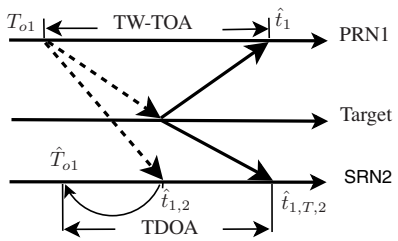

(b)
Fig. 1. (a) A primary node initiates positioning by transmitting a signal to the target whereupon the target replies to the received signal. Both signals are received in the secondary nodes (b) TW-TOA and TDOA for PRN1 and SRN2.

to the SRN as follows (see Fig. 1(b)):

$$
\begin{aligned}
& z_{i}^{j}=c\left(\hat{t}_{i, T, j}-\hat{T}_{o_{i}}\right) \\
& =r_{i}+r_{j}+n_{T, j}+n_{i, T}-n_{i, j}, \quad(i, j) \in \mathcal{C},
\end{aligned}
$$

where $\hat{T}_{o_{i}}$ is an estimate of $T_{o_{i}}$, e.g., $\hat{T}_{o_{i}}=\hat{t}_{i, j}-r_{i, j} / c=$ $T_{o_{i}}+\tilde{n}_{i, j}$, and $n_{i, T}=c \tilde{n}_{i, T}, n_{i, T}=c \tilde{n}_{i, T}$, and $n_{i, j}=c \tilde{n}_{i, j}$. From (1), the distance estimate to the target in the $i$ th PRN is expressed as

$$
z_{i}=c \hat{t}_{i}=r_{i}+\frac{n_{i, T}}{2}+\frac{n_{T, i}}{2}, i=1, \ldots, N .
$$

In the sequel, we consider the vector of measurements $\mathbf{z}$ as follows

$$
\mathbf{z}=\left[\begin{array}{lllllllllll}
z_{1} & \ldots & z_{N} & z_{1}^{1} & \ldots & z_{1}^{M} & \ldots & z_{N}^{1} & \ldots & z_{N}^{M}
\end{array}\right]^{T} .
$$

\section{A. Maximum likelihood estimator algorithm}

It is clear that the vector $\mathbf{z}$ can be modeled as Gaussian random vector $\mathbf{z} \sim \mathcal{N}(\boldsymbol{\mu}, \mathbf{C})$, where mean $\boldsymbol{\mu}=$ $\left[\begin{array}{llllllllll}\mu_{1} & \ldots & \mu_{N} & \mu_{1}^{1} & \ldots & \mu_{1}^{M} & \ldots & \mu_{N}^{1} & \ldots & \mu_{N}^{M}\end{array}\right]^{T}$ and covariance matrix $\mathbf{C}$ are computed as follows ${ }^{2}$ [2],

$$
\begin{aligned}
& \mu_{i}=r_{i}, \quad \mu_{i}^{j}=r_{i}+r_{N+j}, \\
& \mathbf{C}=\mathbb{E}\left\{(\mathbf{z}-\boldsymbol{\mu})(\mathbf{z}-\boldsymbol{\mu})^{T}\right\}=\left[\begin{array}{ll}
\mathbf{C}_{11} & \mathbf{C}_{12} \\
\mathbf{C}_{21} & \mathbf{C}_{22}
\end{array}\right],
\end{aligned}
$$

where matrices $\mathbf{C}_{11} \in \mathbb{R}^{N \times N}, \mathbf{C}_{12}=\mathbf{C}_{21}^{T} \in \mathbb{R}^{N \times N M}$, and $\mathbf{C}_{22} \in \mathbb{R}^{N M \times N M}$ can be obtained as follows

$$
\begin{aligned}
& \mathbf{C}_{11}=\operatorname{diag}\left(\left(\frac{\tilde{\sigma}_{1}^{2}}{4}+\frac{\sigma_{1}^{2}}{4}\right), \ldots,\left(\frac{\tilde{\sigma}_{N}^{2}}{4}+\frac{\sigma_{N}^{2}}{4}\right)\right), \\
& \mathbf{C}_{12}=\left[\begin{array}{ccc}
\mathbf{v}_{1}^{T} & \ldots & \mathbf{0} \\
\vdots & \ddots & \vdots \\
\mathbf{0} & \ldots & \mathbf{v}_{N}^{T}
\end{array}\right], \mathbf{v}_{i}=\frac{\tilde{\sigma}_{i}^{2}}{2} \mathbf{1}_{M}, \\
& \mathbf{C}_{22}=\operatorname{blkdiag}\left(\mathbf{W}_{1}, \ldots, \mathbf{W}_{N}\right), \mathbf{1}_{M}=\left[\begin{array}{lll}
1 & \ldots & 1
\end{array}\right]^{T} \\
& \mathbf{W}_{i}=\tilde{\sigma}_{i}^{2} \mathbf{1}_{M} \mathbf{1}_{M}^{T} \\
& +\operatorname{diag}\left(\sigma_{N+1}^{2}+\sigma_{i, N+1}^{2}, \ldots, \sigma_{N+M}^{2}+\sigma_{i, N+M}^{2}\right) .
\end{aligned}
$$

where (blk) $\operatorname{diag}\left(X_{1}, \ldots, X_{N}\right)$ is a (block) diagonal matrix with diagonal element $X_{1}, \ldots, X_{N}$.

The MLE is obtained by the following optimization problem [5]

$$
\hat{\boldsymbol{\theta}}=\underset{\boldsymbol{\theta} \in \mathbb{R}^{2}}{\operatorname{argmin}}(\mathbf{z}-\boldsymbol{\mu})^{T} \mathbf{C}^{-1}(\mathbf{z}-\boldsymbol{\mu}) .
$$

\footnotetext{
${ }^{2}$ For simplicity of notation, we assume $\sigma_{T, i}=\sigma_{i}$ and $\sigma_{i, T}=\tilde{\sigma}_{i}$.
} 
With some manipulations, (8) can be expressed as [2]

$$
\begin{aligned}
& \hat{\boldsymbol{\theta}}=\underset{\boldsymbol{\theta} \in \mathbb{R}^{2}}{\operatorname{argmin}} \sum_{i=1}^{N}\left\{\left(\frac{2}{\sigma_{i}^{2}}-\frac{1}{s_{i} \sigma_{i}^{4}}\right)\left(z_{i}-r_{i}\right)^{2}\right. \\
& -\frac{1}{s_{i}}\left(\sum_{j=N+1}^{N+M} \frac{z_{i}^{j}-r_{i}-r_{j}}{4 \sigma_{j}^{2}}\right)^{2}-\frac{z_{i}-r_{i}}{s_{i} \sigma_{i}^{2}} \sum_{j=N+1}^{N+M} \frac{\left(z_{i}^{j}-r_{i}-r_{j}\right)}{2 \sigma_{j}^{2}} \\
& \left.+\sum_{j=N+1}^{N+M} \frac{\left(z_{i}^{j}-r_{i}-r_{j}\right)^{2}}{2 \sigma_{j}^{2}}\right\}
\end{aligned}
$$

where $s_{i} \triangleq 1 /\left(2 \tilde{\sigma}_{i}^{2}\right)+1 /\left(2 \sigma_{i}^{2}\right)+\sum_{j=N+1}^{N+M} 1 /\left(4 \sigma_{j}^{2}\right)$. As can be seen, there is no closed-form solution for the ML estimate and we are forced to use numerical method, e.g., iterative search with good initial point. Note that for the conventional network, i.e., a network consisting of only primary nodes, MLE changes to [2]

$$
\hat{\boldsymbol{\theta}}=\underset{\boldsymbol{\theta} \in \mathbb{R}^{2}}{\operatorname{argmin}} \sum_{i=1}^{N} \frac{2}{\sigma_{i}^{2}}\left(z_{i}-r_{i}\right)^{2} .
$$

\section{B. Linear estimator algorithm}

To obtain a closed-form solution to the positioning problem, which avoids MLE's drawback, we apply linearization technique to the measurements in both PRNs and SRNs. The estimator is implemented in two steps; coarse and fine. Note that it is required to have at least three non co-linear reference nodes for this estimator.

1) Coarse estimation: One way to obtain a linear model versus the target position is to apply a pre-processing on measurements. Suppose that the level of noise is small, for the $i$ th PRN, squaring both sides of (5) yields

$$
\begin{aligned}
& z_{i}^{2} \approx\|\boldsymbol{\theta}\|^{2}-2 \mathbf{x}_{i}^{T} \boldsymbol{\theta}+\left\|\mathbf{x}_{i}\right\|^{2}+2 r_{i} \vartheta_{i} \\
& \quad=\left[\begin{array}{ll}
-2 \mathbf{x}_{i}^{T} & 1
\end{array}\right] \boldsymbol{\psi}+\left\|\mathbf{x}_{i}\right\|^{2}+2 r_{i} \vartheta_{i}, i=1, \ldots, N,
\end{aligned}
$$

where $\vartheta_{i}=n_{i, T} / 2+n_{T, i} / 2$ and $\boldsymbol{\psi}=\left[\boldsymbol{\theta}^{T}\|\boldsymbol{\theta}\|^{2}\right]^{T}$. For simplicity of notation let us define

$$
\bar{z}_{i}=z_{i}^{2}-\left\|\mathbf{x}_{i}\right\|^{2}=\left[-2 \mathbf{x}_{i}^{T} 1\right] \boldsymbol{\psi}+2 r_{i} \vartheta_{i}, i=1, \ldots, N .
$$

For the TDOA measurement in the $j$ th SRN, i.e., (4), let us first arrange a new set of measurements as follows

$$
\tilde{z}_{i}^{j}=z_{i}^{j}-z_{i}=r_{j}+\epsilon_{i, j},(i, j) \in \mathcal{C},
$$

where $\epsilon_{i, j}=n_{T, j}+n_{i, T} / 2-n_{T, i} / 2-n_{i, j}$. Now similar to (11), we can linearize (12) to get

$$
\left(\tilde{z}_{i}^{j}\right)^{2} \approx\left[-2 \mathbf{x}_{j}^{T} 1\right] \boldsymbol{\psi}+\left\|\mathbf{x}_{j}\right\|^{2}+2 r_{j} \epsilon_{i, j}, \quad(i, j) \in \mathcal{C} .
$$

Again for simplicity of notation let us define

$$
\bar{z}_{i}^{j}=\left(\tilde{z}_{i}^{j}\right)^{2}-\left\|\mathbf{x}_{j}\right\|^{2}=\left[\begin{array}{ll}
-2 \mathbf{x}_{j}^{T} & 1
\end{array}\right] \boldsymbol{\psi}+2 r_{j} \epsilon_{i, j}, \quad(i, j) \in \mathcal{C} .
$$

A linear set of equations can be written as

$$
\mathbf{d}=\mathbf{A} \psi+\nu
$$

where vectors $\mathbf{d}$ and $\boldsymbol{\nu}$, and matrix $\mathbf{A}$ are obtained as follows

$$
\begin{aligned}
& \mathbf{d}=\left[\begin{array}{llllll}
\bar{z}_{1} & \ldots & \bar{z}_{N} & \bar{z}_{1}^{1} & \ldots & \bar{z}_{1}^{N}
\end{array}\right]^{T}, \\
& \boldsymbol{\nu}=2\left[\begin{array}{llllll}
r_{1} \vartheta_{1} & \ldots & r_{N} \vartheta_{N} & r_{N+1} \epsilon_{i, N+1} & \ldots & r_{N+M} \\
\epsilon_{i, N+M}
\end{array}\right]^{T} \text {, } \\
& \mathbf{A}=\left[\begin{array}{c}
\mathbf{A}_{1} \\
\mathbf{A}_{2} \\
\vdots \\
\mathbf{A}_{2}
\end{array}\right], \mathbf{A}_{1}=\left[\begin{array}{cc}
-2 \mathbf{x}_{1}^{T} & 1 \\
\vdots & \vdots \\
-2 \mathbf{x}_{N}^{T} & 1
\end{array}\right], \mathbf{A}_{2}=\left[\begin{array}{cc}
-2 \mathbf{x}_{N+1}^{T} & 1 \\
\vdots & \vdots \\
-2 \mathbf{x}_{N+M}^{T} & 1
\end{array}\right] \text {, }
\end{aligned}
$$

Suppose that the matrix $\mathbf{A}$ is full-rank, then the unknown parameter $\psi$ can be estimated by using weighted least squares method [5, Ch. 8],

$$
\hat{\boldsymbol{\psi}}=\left(\mathbf{A}^{T} \mathbf{C}_{\boldsymbol{\nu}}^{-1} \mathbf{A}\right)^{-1} \mathbf{A}^{T} \mathbf{C}_{\boldsymbol{\nu}}^{-1} \mathbf{d},
$$

where the covariance matrix $\mathbf{C}_{\nu}$ of the zeros mean noise vector $\nu$ is computed as follows

$$
\mathbf{C}_{\boldsymbol{\nu}}=\mathbb{E}\left\{\boldsymbol{\nu} \boldsymbol{\nu}^{T}\right\}=\left[\begin{array}{ll}
\mathbf{C}_{\boldsymbol{\nu}_{11}} & \mathbf{C}_{\boldsymbol{\nu}_{12}} \\
\mathbf{C}_{\boldsymbol{\nu}_{21}} & \mathbf{C}_{\boldsymbol{\nu}_{22}}
\end{array}\right],
$$

where matrices $\mathbf{C}_{\boldsymbol{\nu}_{11}} \in \mathbb{R}^{N \times N}, \mathbf{C}_{\boldsymbol{\nu}_{12}}=\mathbf{C}_{\boldsymbol{\nu}_{21}}^{T} \in \mathbb{R}^{N \times N M}$, and $\mathbf{C}_{\boldsymbol{\nu}_{22}} \in \mathbb{R}^{N M \times N M}$ are given by

$$
\begin{aligned}
& \mathbf{C}_{\boldsymbol{\nu}_{11}}=\operatorname{diag}\left(r_{1}^{2}\left(\tilde{\sigma}_{1}^{2}+\sigma_{1}^{2}\right), \ldots, r_{N}^{2}\left(\tilde{\sigma}_{N}^{2}+\sigma_{N}^{2}\right)\right), \\
& \mathbf{C}_{\boldsymbol{\nu}_{12}}=\left[\begin{array}{ccc}
\mathbf{r}_{1}^{T} & \ldots & \mathbf{0} \\
\vdots & \ddots & \vdots \\
\mathbf{0} & \ldots & \mathbf{r}_{N}^{T}
\end{array}\right], \mathbf{C}_{\boldsymbol{\nu}_{22}}=\left[\begin{array}{ccc}
\mathbf{R}_{1} & \ldots & \mathbf{0} \\
\vdots & \ddots & \vdots \\
\mathbf{0} & \ldots & \mathbf{R}_{N}
\end{array}\right], \\
& \mathbf{r}_{i}=r_{i}\left[r_{N+i}\left(\tilde{\sigma}_{i}^{2}+\sigma_{i}^{2}\right) \ldots r_{N+M}\left(\tilde{\sigma}_{i}^{2}+\sigma_{i}^{2}\right)\right]^{T}, \\
& \mathbf{R}_{i}=\left(\tilde{\sigma}_{i}^{2}+\sigma_{i}^{2}\right)\left[\begin{array}{c}
r_{N+1} \\
\vdots \\
r_{N+M}
\end{array}\right]\left[r_{N+1} \ldots r_{N+M}\right]+4 \operatorname{diag}( \\
& \left.r_{N+1}^{2}\left(\sigma_{N+1}^{2}+\tilde{\sigma}_{N+1}^{2}\right), \ldots, r_{N+M}^{2}\left(\sigma_{N+M}^{2}+\tilde{\sigma}_{N+M}^{2}\right)\right),(17)
\end{aligned}
$$

where $\mathbf{I}_{M}$ is the $M$ by $M$ identity matrix. The covariance matrix of $\hat{\psi}$ is given by [5]

$$
\operatorname{cov}(\hat{\boldsymbol{\psi}})=\left(\mathbf{A}^{T} \mathbf{C}_{\boldsymbol{\nu}}^{-1} \mathbf{A}\right)^{-1}
$$

To compute the covariance matrix $\mathbf{C}_{\boldsymbol{\nu}}$ the real distances between reference nodes to the target are required. Since in practice, the real distances are not available, we instead use the measured distances. It has been shown in [6] that the degradation of replacing the estimated distances instead of the real distances is negligible. The linear estimator obtained in (15) shows good performance when the measurement noise is small. The first step estimation gives a coarse estimate and to improve it we obtain a refining estimator.

2) Fine estimation: Let us apply the first order Taylor-series expansion about $\hat{\boldsymbol{\theta}}=[\hat{\boldsymbol{\psi}}]_{2 \times 1}$ for the $i$ th measurement in the PRNs, i.e., (5), to get

$$
z_{i}=r_{i}+\nabla^{T} r_{\left.i\right|_{\hat{\boldsymbol{\theta}}}}(\boldsymbol{\theta}-\hat{\boldsymbol{\theta}})+\vartheta_{i}
$$

where the gradient is computed as $\nabla^{T} r_{\left.i\right|_{\hat{\boldsymbol{\theta}}}}=(\hat{\boldsymbol{\theta}}-$ $\left.\mathbf{x}_{i}\right)^{T} /\left\|\hat{\boldsymbol{\theta}}-\mathbf{x}_{i}\right\|$. 
For the measurement in the SRN similar to measurement in PRN, we get

$$
\begin{aligned}
& z_{i}^{j}=r_{\left.i\right|_{\hat{\boldsymbol{\theta}}}}+r_{\left.j\right|_{\hat{\boldsymbol{\theta}}}}+\nabla^{T} r_{\left.i\right|_{\hat{\boldsymbol{\theta}}}}(\boldsymbol{\theta}-\hat{\boldsymbol{\theta}})+\nabla^{T} r_{\left.j\right|_{\hat{\boldsymbol{\theta}}}}(\boldsymbol{\theta}-\hat{\boldsymbol{\theta}})+\vartheta_{i}^{j}, \\
& (i, j) \in \mathcal{C},
\end{aligned}
$$

where $\vartheta_{i}^{j}=n_{T, j}+n_{i, T}-n_{i, j}$. A new linear set of data can be written in matrix form as

$$
\mathbf{h}=\mathbf{G} \triangle \boldsymbol{\theta}+\boldsymbol{\zeta}
$$

where $\triangle \boldsymbol{\theta}=\boldsymbol{\theta}-\hat{\boldsymbol{\theta}}$ and vectors $\mathbf{h}, \boldsymbol{\zeta}$, and matrix $\mathbf{G}$ are obtained as follows

$$
\begin{aligned}
& \mathbf{h}=\left[\begin{array}{llllll}
z_{1} & \ldots & z_{N} & z_{1}^{1} & \ldots & z_{N}^{M}
\end{array}\right]^{T}, \\
& \mathbf{G}=\left[\begin{array}{c}
\nabla^{T} r_{\left.1\right|_{\hat{\boldsymbol{\theta}}}} \\
\vdots \\
\nabla^{T} r_{\left.N\right|_{\hat{\boldsymbol{\theta}}}} \\
\nabla^{T} r_{\left.1\right|_{\hat{\boldsymbol{\theta}}}}+\nabla^{T} r_{N+\left.1\right|_{\hat{\boldsymbol{\theta}}}} \\
\vdots \\
\nabla^{T} r_{\left.N\right|_{\hat{\boldsymbol{\theta}}}}+\nabla^{T} r_{N+\left.M\right|_{\hat{\boldsymbol{\theta}}}}
\end{array}\right] \\
& \boldsymbol{\zeta}=\left[\begin{array}{llllll}
\vartheta_{1} & \ldots & \vartheta_{N} & \vartheta_{1}^{N+1} & \ldots & \vartheta_{N}^{N+M}
\end{array}\right]^{T} \text {. }
\end{aligned}
$$

To obtain the estimation error from (21), i.e. $\triangle \boldsymbol{\theta}$, to be small enough (if possible), we solve a regularized least squares problem as follows

$$
\underset{\triangle \boldsymbol{\theta}}{\operatorname{minimize}}\|\mathbf{h}-\mathbf{G} \triangle \boldsymbol{\theta}\|_{\mathbf{C}^{-1}}^{2}+\gamma\|\triangle \boldsymbol{\theta}\|^{2}
$$

where regularization parameter $\gamma>0$ determines the tradeoff between $\|\mathbf{h}-\mathbf{G} \triangle \boldsymbol{\theta}\|^{2}$ and $\|\triangle \boldsymbol{\theta}\|^{2}$, and $\|\mathbf{b}\|_{\mathbf{P}}^{2}$ stands for the weighted norm $\mathbf{b}^{T} \mathbf{P b}$. The solution to (22), Tikhonov regularization problem, is given by $[7$, Ch. 6]

$$
\hat{\triangle} \boldsymbol{\theta}=\left(\mathbf{G}^{T} \mathbf{C}^{-1} \mathbf{G}+\gamma \mathbf{I}_{2}\right)^{-1} \mathbf{G}^{T} \mathbf{C}^{-1} \mathbf{h}
$$

where the covariance matrix of $\mathbf{C}^{-1}$ is given by (7).

The final estimate is expressed as

$$
\tilde{\boldsymbol{\theta}}=\hat{\boldsymbol{\theta}}+\hat{\triangle} \boldsymbol{\theta}
$$

Eq.(23) shows that the estimator is a biased estimator. It is easy to show that for high SNR the estimator is an unbiased estimator. In the simulation section, we examine the bias value of the estimator.

As can be seen from (24), the estimator in the second step tries to refine the previous estimate. Normally the second step can be repeated in order to improve the accuracy, but as we have observed in simulations, at high SNR, one step fine tuning is enough to get very close to the CRLB.

Note that the method explained here can be similarly applied to non-cooperative networks.

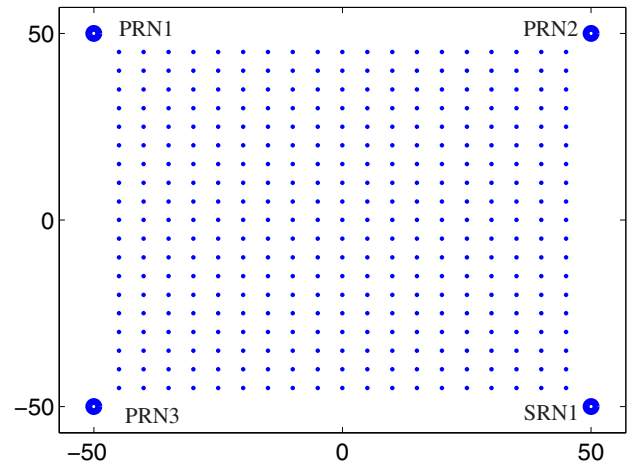

Fig. 2. Simulation environment with three primary nodes and one secondary node. The coordinates are in the unit of meters.

\section{SIMULATION}

In this section, computer simulations are performed to evaluate the performance of the proposed estimator. To compare different methods, we consider root mean-square error (RMSE) and cumulative distribution function (CDF) of positioning error. The CRLB for the cooperative case computed in [2] is re-derived in appendix A. Note that the CRLB for non-cooperative mode can be easily obtained from the cooperative case neglecting the secondary nodes' contribution. Fig. 2 shows the simulation environment where three PRNs and one SRN involved in simulation are located on the corner of a $100 \mathrm{~m} \times 100 \mathrm{~m}$ square area. The target is randomly placed inside of the area over a grid of $19 \times 19$. In simulation, we assume $\tilde{\sigma}_{i}=\sigma_{i}=\sigma_{i, j}=\sigma$. For implementation of the MLE, we used grid search inside the area. For the fine estimator without any attempt to optimize $\gamma$, we simply set it equal to 0.01 for both conventional and cooperative network.

Fig. 3 shows the RMSE of the CRLB and the proposed estimator for both cooperative and non-cooperative networks. As can be seen, for both networks, the linear estimator, as well as MLE, attains the CRLB for high SNR. As the variance of noise increases, the gap between proposed estimator and the CRLB also increases. It also shows that the cooperation can improve the performance for low SNRs. It is seen that the fine estimator outperforms the coarse estimator for both networks. For this deployment, it is seen that the coarse estimator for the cooperative case outperforms the optimal unbiased estimator in the non-cooperative case. To further evaluation, we consider the position error CDF for the linear estimators in both cooperative and non-cooperative modes. In this simulation, we consider the network of Fig. 2 and set $\sigma_{T}=\sigma_{i}=10 \mathrm{~m}$. Fig. 4 shows the position error $\mathrm{CDF}$ for both cases. It can be seen that in the cooperative mode the proposed estimator outperforms that one in the non-cooperative mode. It also shows that the fine estimator improves the estimation accuracy compared to the coarse estimator for both networks. We further study the bias value of proposed estimator. To evaluate the bias of the estimator, we compute the root square of mean biased value (RSMB) which we define as $\mathrm{RSMB}=\sqrt{\left[\mathrm{E}\left\{\hat{\theta}_{1}-\theta_{1}\right\}\right]^{2}+\left[\mathrm{E}\left\{\hat{\theta}_{2}-\theta_{2}\right\}\right]^{2}}$. 


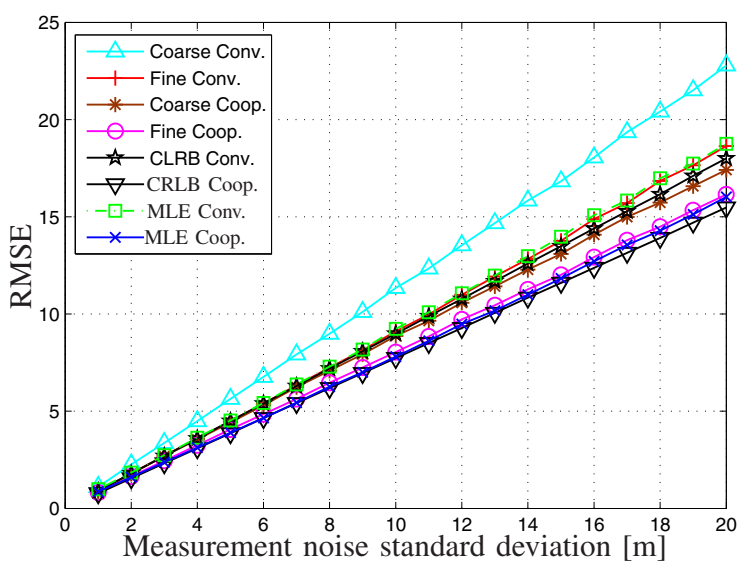

Fig. 3. RMSE of the CRLB, MLE, and the linear estimators for both cooperative and non-cooperative cases.

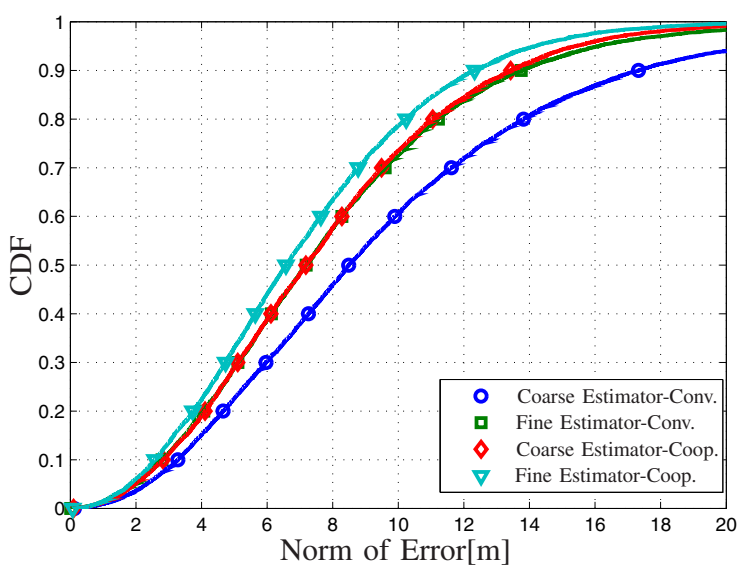

Fig. 4. CDF of the linear estimators for both cooperative and non-cooperative networks $(\sigma=10 \mathrm{~m})$.

Fig. 5 shows the RSMB of estimator for both conventional and cooperative networks. As can be seen in cooperative mode the bias value is extremely small compared to CRLB. Hence the fine estimator for cooperative mode can be considered as an unbiased estimator.

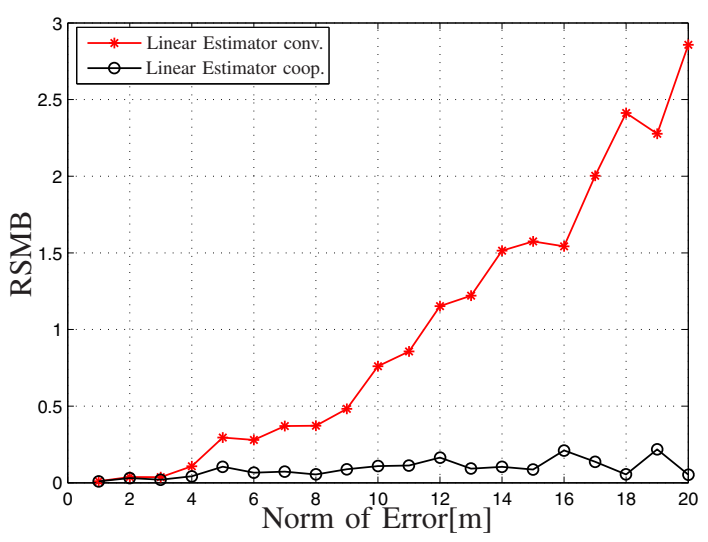

Fig. 5. RSMB of the linear estimators for both cooperative and noncooperative networks.

\section{CONCLUSION}

In this paper, we considered the positioning problem in cooperative sensor networks where hybrid two-way time of arrival and time difference of arrival are measured by senor nodes. Based on measurements collected by different nodes, a maximum likelihood estimator (MLE) can be derived. Due to the non-linear nature of the cost function in the MLE, a numerical method, e.g., an iterative search algorithm with a good initial point, should be taken. To avoid drawbacks in the numerical method, we have used the linearization techniques to obtain a two-step linear estimator. In the first step, a coarse estimate is obtained and in the second step, it is refined. The advantages of the proposed estimator are that it has a closedform solution in each step and also it attains the CarmérRao lower bound for sufficiently high SNR. Simulation results show that the proposed method is asymptotically efficient.

\section{APPENDIX}

\section{A. $C R L B$}

Considering the measurement vector (6) with mean $\boldsymbol{\mu}$ and covariance matrix $\mathbf{C}$, i.e., (7), the fisher information matrix can be computed as [5] $[I]_{n m}=\left[\frac{\partial \boldsymbol{\mu}}{\partial \theta_{n}}\right]^{T} \mathbf{C}^{-1}\left[\frac{\partial \boldsymbol{\mu}}{\partial \theta_{m}}\right], n=$ $1,2, m=1,2$. Simple calculation considering $\boldsymbol{\theta}=\left[\begin{array}{ll}\theta_{1} & \theta_{2}\end{array}\right]^{T}$ shows

$$
\begin{aligned}
& {\left[\frac{\partial \boldsymbol{\mu}}{\partial \theta_{n}}\right]^{T}=\left[\frac{\partial \mu_{1}}{\partial \theta_{n}} \ldots \frac{\partial \mu_{N}}{\partial \theta_{n}} \ldots \frac{\partial \mu_{N}^{1}}{\partial \theta_{n}} \ldots \frac{\partial \mu_{N}^{M}}{\partial \theta_{n}}\right],} \\
& \frac{\partial \mu_{i}}{\partial \theta_{1}}=\frac{x-x_{i}}{r_{i}}, \frac{\partial \mu_{i}^{j}}{\partial \theta_{1}}=\frac{x-x_{i}}{r_{i}}+\frac{x-x_{N+j}}{r_{N+j}},(i, j) \in \mathcal{C}, \\
& \frac{\partial \mu_{i}}{\partial \theta_{2}}=\frac{y-y_{i}}{r_{i}}, \frac{\partial \mu_{i}^{j}}{\partial \theta_{2}}=\frac{y-y_{i}}{r_{i}}+\frac{y-y_{N+j}}{r_{N+j}},(i, j) \in \mathcal{C} .
\end{aligned}
$$

The lower bound on any unbiased estimator is given by

$$
E\left\{\|\hat{\boldsymbol{\theta}}-\boldsymbol{\theta}\|^{2}\right\} \geq \frac{[I]_{22}+[I]_{11}}{[I]_{11}[I]_{22}-[I]_{12}^{2}}
$$

\section{REFERENCES}

[1] A. Sayed, A. Tarighat, and N. Khajehnouri, "Network-based wireless location: challenges faced in developing techniques for accurate wireless location information," IEEE Signal Processing Magazine, vol. 22, no. 4, pp. 24-40, July 2005.

[2] S. Gezici and Z. Sahinoglu, "Enhanced position estimation via node cooperation," in Proc. IEEE International Conference on Communications (ICC), Cape Town, South Africa, May 23-27, 2010.

[3] R. Fujiwara, K. Mizugaki, T. Nakagawa, D. Maeda, and M. Miyazaki, "TOA/TDOA hybrid relative positioning system using UWB-IR," in IEEE Radio and Wireless Week, January 2009, pp. 679-682.

[4] H. Wymeersch, J. Lien, and M. Z. Win, "Cooperative localization in wireless networks," Proceedings of the IEEE, vol. 97, no. 2, pp. 427450, February 2009.

[5] S. Kay, Fundamentals of statistical Signal Processing: Estimation Theory. Upper Saddle River, NJ: Prentice-Hall, 1993.

[6] K. C. Ho, X. Lu, and L. Kovavisaruch, "Source localization using TDOA and FDOA measurements in the presence of receiver location errors: analysis and solution," IEEE Transactions on Signal Processing, vol. 55, no. 2, pp. $684-696$, February 2007.

[7] S. Boyd and L. Vandenberghe, Convex Optimization. Cambridge University Press, 2004 